\title{
ELECTROELASTIC STATE OF AN INHOMOGENEOUS PIEZOCERAMIC LAYER UNDER SYMMETRIC LOADING
}

\author{
Yu. D. Kovalev* and E. N. Stativka
}

Keywords: piezoceramic layer, electroelasticity, symmetric loading, cylindrical inclusion

A procedure for solving three-dimensional mixed symmetric problems of electroelasticity theory for a piezoceramic layer with an inclusion, which is weakened with a through hole, is proposed. The boundary-value problem is reduced to a system of $12 k(k=1,2, \ldots)$ integrodifferential equations. Expressions for stresses characterizing the stress state of the inhomogeneous layer are found. Calculation results for characteristic stresses are presented.

Due to its unique properties, piezoceramics recently have increasingly been used in various branches of engineering. The steady widening of the field of application of piezoceramic materials necessitates a further profound investigation into the laws of static deformation of structural elements made of such materials.

The stress state of a transtropic (isotropic) layer weakened by through tunnel holes at sliding fixation of its end faces (symmetric case) is examined in [1-3]. In these studies, the solutions of boundary-value problems are constructed by using the half-inverted Vorovich method. The bending of an isotropic layer (half-layer) weakened by a noncircular through hole is considered in [4]. A similar problem for an isotropic layer with a circular hole is solved in [5, 6] by other methods. In [7], some electroelasticity problems for a layer under different boundary conditions are considered. The stress state of hollow cylinders of finite length is examined in [8,9]. In [10-13], various methods for solving three-dimensional problems of elasticity and electroelasticity theories are suggested. A general approach to the solution of mixed problems of elasticity and electroelasticity theories for a layer weakened by through tunnel inhomogeneities, distinct from that described in [3], is offered in [14]. This approach was used to consider a mixed axisymmetric problem of electroelasticity for a piezoceramic layer weakened by a through hole [15], to examine an axisymmetric problem for an inhomogeneous piezoceramic layer [16], to solve an axisymmetric problem for an inhomogeneous piezoceramic cylinder [17], and to investigate the stress state of an inhomogeneous piezoceramic cylinder under symmetric loading [18].

The Sumy State University, Ukraine

*Corresponding author; e-mail: leonid@mphis.sumdu.edu

Translated from Mekhanika Kompozitnykh Materialov, Vol. 47, No. 5, pp. 793-806, September-October, 2011. Original article submitted March 2, 2011. 